23rd.-Has passed a more comfortable night: the conjunctivæ less suffused; countenance more intelligent; tongue becoming moist and less red; pulse ninety-four; bowels constipated. Ordered, one drop of croton oil, every four hours; chloroform repeated.-Evening: appears better in every respect; the bowels have acted. Ordered, muriate of morphia, one grain, to be taken immediately; and chloroform.

24th.-Had several hours sleep, and is more sensible; complains of his hands and feet feeling cold. The ice to be discontinued, and to have hot water to the feet. Ordered, chloric ether, half an ounce, carbonate of ammonia half a drachm, camphor mixture seven ounces and a half. Mix. An ounce to be taken every three hours.-Evening: better in every respect, except that the pulse has got up to 120 ; this might be attributed to his having had too many of his friends seeing him. Gave the chloroform, and in fifteen minutes we found the pulse had come down to eighty-eight. Repeat the morphia and mixture.

25th.- Has had an excellent night; returning intelligence; pulse ninety-four; skin warm; still passes his urine and stools under him; expression of countenance improving. From this time the improvement was so rapid and continuous that $I$ shall not consider it necessary to describe the symptoms daily. The chloroform was administered occasionally, with tonics and stimulants; and in about a fortnight he went into the country, quite well both in mind and body.

Liverpool, 1848.

\section{ON A CASE OF}

ABSCESS OF THE THORACIC WALLS, SIMULATING PLEURITIS AND EMPYEMA.

BY HENRY CRISP, M.B. Lond.

The following case occurred in the practice of my friend Mr. Martin, of Haverhill, Suffolk, who kindly gave me an opportunity of watching the patient throughout. It is interesting, from its similitude to an attack of pleuritis, under which disease, indeed, the patient was at first supposed to be labouring. It has also some points of analogy with the case reported in the number of The LANCET for July 1st, p. 16, by Dr. Macdonnell, of Montreal.

On the first appearance of the swelling it might have been supposed that there was a collection of pus in the pleura, which was making its way externally; but there were no stethoscopic signs which would indicate the presence of empyema.

J.S-, twenty-two years of age, cabinet-maker, of rather intemperate habits, was attacked, in February, 1848, with pain of a sharp, stabbing character in the right side; he was of unhealthy appearance, and one or two members of his family had died of phthisis. The pain was aggravated by making pressure over and between the ribs, by taking a full inspiration, by coughing, and by any motion of the affected side. There was no audible "rubbing sound," nor dulness on percussion. Large crepitation could be heard on both sides of the chest, and the respiratory murmur was present in all parts of the lungs, though very feeble. He had a slight cough, and expectorated a moderate quantity of transparent, viscid mucus; respiration was slightly quickened, and the intercostal muscles of the affected side did not act so freely as those of the other side. These symptoms had been preceded by slight shivering; pulse-about 80 , small and weak; tongue furred; bowels confined; and he had slight thirst. He was ordered aperient medicines, with small doses of tartar-emetic every five hours, and a mild mercurial at night. This treatment was continued for some time, the patient occasionally feeling better, but never entirely losing the pain. The cough still continued, but he was not confined to his bed; his tongue was always much furred, and great difficulty was experienced in getting his bowels to act properly. His pulse was, for the most part, quicker than natural; his appetite was variable, but generally good.

About three weeks from the appearance of these symptoms, he first noticed a swelling over the site of the pain, just below the right mamma; this was boggy and diffusednot very painful on pressure. At first there was little redness, but after a short time the integuments became of a pinkish hue. 'There was no dulness of the affected side; the respiratory murmur, though very faint on both sides, was audible all over the chest; pulse about 90 , weak; bowels constipatedmotions very dark-coloured; his cough still troubles him, and much increases the pain in the side.

Abont a week after this he had rigors; the swelling became softer, and matter was detected; though this was at first deep- eated, pressure did not empty the sac. Leeches and hot fomentations were employed when the swelling first made its ppearance, and appropriate medicines given internally. $\mathbf{H e}$ became very low; the pulse was quick, weak, and irritable; his tongue was covered with a thick dark fur, and he lost his appetite; the pain was so severe as to prevent his sleeping. Poultices were applied for a few days, and then the abscess was opened. About a teacupful of white, inodorous pus scaped.

Poultices were continued; ammonia and wine, and afterards quinine, were given internally.

He was very much relieved by the evacuation of the abscess: he slept better, his appetite returned, and he lost his cough entirely. In two or three days, the wound, which had discharged a little, entirely closed, and he gradually but perfectly recovered his former health.

London, July, 1848.

\section{ON PYROGEN-ONLY ONE ELECT'RIC FLUID- MAGNETISM A PROPERTY OF PYROGEN.}

\section{BY JOHN J. LAKE, Esq., Portsmouth.}

IN my last communication on this subject, (THE LANCET, April 22nd, p. 447,) it is stated that potassium was first obtained by the withdrawal of pyrogen from moistened hydrate of potash. This is assuming that there is only one kind of fluid, which I have designated pyrogen, and not two, according to the now commonly adopted hypothesis.

In advocating the old but neglected theory of Watson, Franklin, and other fathers of this branch of science, it will be necessary to answer some objections that have been advanced with effect against it; and this will lead to the consideration of some difficulties in the way of adopting the opposite theory of two fluids. For instance, it is received that two pith balls, suspended by silk threads, when in a negative state, mutually " repel each other;" and it has been deemed impossible to account for the absence of fluid producing such an effect. This, the usual manner of describing the visible appearances, is greatly calculated to conceal the real cause. Two balls, thus suspended, and in a negative state, do not repel each other. They diverge from a common point, but exert upon each other no particular pyrogenic influence. This point is the centre of a pyrogenic vacuum, (not a vacuum of all matter, but a deficiency of pyrogen, ) of greater or less extent, created in the atmosphere, as well as on the balls, owing to the latter attracting the portion of pyrogen in their immediate neighbourhood. The fluid without this vacuum necessarily endeavours to fill it up, and in seeking to do so, draws the pith halls towards it, as a ready medium of transport, the lightness of the pith enabling it to do so. In like manner, when they are charged to excess with pyrogen, the fluid, seeking to diffuse itself, and restore the equilibrium, carries them outwards along with it, and for the same reason.

Again, when a card, or pack of cards, or folds of paper, are pierced by a discharge of pyrogen, there is a mark observed by the side of the hole, indicating a current in the opposite direction to that discharged, but parallel with it. This is usually viewed as proving the existence of a current of negative fluid. It is not, however, by any means a sure sign of the motion of two fluids, because, first, supposing two fluids, they should move with equal force, and each make a complete perforation; secondly, on the same supposition, they ought not to move parallel at all, but take the shortest path to meet and unite with each other, and so restore their equilibrium; for on this hypothesis " the particles of each fluid are conceived to exert upon each other a strong repulsive force; while particles of the unlike fluids mutually attract each other." And it is difficult to imagine how it can be supposed, that on completing the circuit of a battery the positive and negative fluids are compelled, by acting against each other, to move spirally about the wire, since the mutual attraction they are assumed to have for each other should effect a combination, and thus stay their progress.*

* On the theory of two fluids, it would be impossible to construct a galvanic battery of more than one pair of plates. Beyond this, each plate would neutralize the effect produced by the one in the adjoining division of the trough to which it is united; for, supposing each to produce an equal quantity of its particular fluid, the one would be absorbed by the other as fast as generated. This, however, is contrary to experience, for the quantity of fluid generated in a battery is in proporition to the number and size of the plates. Then, on completing the circuit, the attraction supposed to exist between the two fluids should stop their progress, and they should combine with each other, and not make a continual circuit in opposite directions, opposing each other so strongly as to cause each to deviate from with the hypothesis of one fluid. 